\title{
In vitro assessment of equipment and software to assess tidal breathing parameters in infants
}

\author{
G. Schmalisch*, B. Foitzik*, R.R. Wauer*, J. Stocks\#
}

In vitro assessment of equipment and software to assess tidal breathing parameters in infants. G. Schmalisch, B. Foitzik, R. R. Wauer, J. Stocks. (C)ERS Journals Ltd 2001.

ABSTRACT: The aim of this in vitro study was to compare the measurement accuracy of two currently available devices for measuring tidal breathing in infants.

A mechanical model pump was used to generate flow profiles which simulated those observed in infants. A range of flows was applied simultaneously to two different devices, namely the commercially available SensorMedics 2600 (SM 2600) and more recently developed, custom-made equipment based on the flow-through technique (FTT). Automatically derived values from both devices were compared with one another and with manual calculations of printouts of the same breaths.

There were no differences in the raw flow signal obtained from the two devices, nor between values calculated automatically or manually from the FTT. Similarly, the deviations between the FTT and SM 2600 were $<3 \%$ for tidal volume, respiratory frequency and minute ventilation. However, when comparing either with manually calculated values or those derived automatically from the FTT, there was a systematic and highly significant underestimation of shape-dependent parameters, such as the time to peak tidal expiratory flow as a proportion of tidal expiratory time ( $t$ PTEF/tE), derived by the SM 2600. The lower the applied flow, the higher the observed deviations, the underestimation being up to $60 \%$ when flows simulating those observed in preterm neonates were applied.

These errors appear to result from differences in signal processing such as the algorithms used for breath detection and can only be detected if appropriate nonsinusoidal flow profiles representing those seen in infants are used to evaluate equipment. Eur Respir J 2001; 17: 100-107.
*Dept of Neonatology (Charité), Humboldt University Berlin, Germany and \#Portex Anaesthesia, Intensive Therapy and Respiratory Medicine Unit, Institute of Child Health, London, UK.

Correspondence: G. Schmalisch, Dept of Neonatology (Charité), Humboldt University Berlin, Schumannstraße 20/ 21, D-10098, Berlin, Germany.

Fax: 493028025824

Keywords: Equipment, flow-through technique, infants, respiratory function tests, software, validation

Received: June 301999

Accepted after revision July 262000

This work, was supported by the German Ministry for Research and Technology, project "Perinatal Lung" (grant 01 ZZ 9511) and Deutsche Forschungsgemeinschaft (Schm 1160/ $1-2)$.
In contrast to that used in adults, in vivo validation of equipment designed to measure respiratory function in infants is extremely difficult, due to the highly variable breathing pattern, limited period of sleep when recordings can be obtained and the potential influence of apparatus dead space on the recordings achieved [1]. Consequently, greater reliance must be placed on in vitro evaluation in this age group. Unfortunately, in the past, such evaluations have often been performed with relatively large sinusoidal signals that do not necessarily represent the variety of flow signals that are observed during infant respiratory function tests (RFTs). Although several in vitro studies have been performed to assess precision of various infant RFT devices [2-5], a thorough assessment of measurement error has been difficult due to the wide range of signals encountered in this age group according to age, clinical condition and type of test. Thus while peak flow may be less than $30 \mathrm{~mL} \cdot \mathrm{s}^{-1}$ during tidal breathing in a sick preterm infant, it may exceed $2 \mathrm{~L} \cdot \mathrm{s}^{-1}$ during forced expiratory manoeuvres from raised lung volume in a healthy 18 month old. A European Respiratory Society/ American Thoracic Society (ERS/ATS) task force is trying to address some of these problems by defining minimal performance criteria for equipment [6] and software [7].

During the last few years, several new techniques have been developed [8] and measurements are now more commonly performed with commercially available equipment than in the past. There is an urgent need for careful validation of such devices by in vitro measurements prior to any clinical or research applications. While the accuracy of volume measurements can easily be determined using calibration syringes [5], the accuracy of other tidal breathing parameters is more difficult to determine, since this requires a model pump with adjustable flow profiles if it is to provide a meaningful simulation of in vivo measurements [9]. With increasing emphasis on the need for quality control and standardization of infant RFTs if these are to be used as reliable outcome measures in clinical or research studies, it is essential to compare the performance of different devices under conditions that closely resemble those likely to be encountered physiologically.

The aim of this study was to use a model pump that could deliver a variety of tidal volumes with variable flow profiles to compare the measurement accuracy of 
two currently available devices for measuring tidal breathing in infants.

\section{Methods}

\section{Model pump}

A mechanical model pump (fig. 1) was used to generate flow profiles which would simulate those observed in infants. As shown in figure 1 the pump delivered the flow profiles via a T-piece to both devices in the same circuit, simultaneously. Airflow was generated by a motor driven pump which consisted of a spring loaded silicon bellow connected to an exchangeable cam disk. During inspiration, the connecting rod was held at point A (fig. 1), resulting in a relatively sinusoidal flow pattern. At the beginning of expiration the holding point of the connecting rod moved from point $\mathrm{A}$ to point $\mathrm{B}$, thereby changing the expiratory flow pattern. At the end of expiration the holding point moved back from $\mathrm{B}$ to $\mathrm{A}$.

The resistance of the model pump was sufficiently high $\left(21 \mathrm{kPa} \cdot \mathrm{L}^{-1} \cdot \mathrm{s}\right.$ at $\left.5 \mathrm{~L} \cdot \mathrm{min}^{-1}\right)$ to ensure that the additional small resistance of the PNTs and tubing did not impair the flow profile generated. By exchanging the silicon bellow and cam disk, flow signals with a volume of $15 \mathrm{~mL}, 29 \mathrm{~mL}, 46 \mathrm{~mL}$ or $61 \mathrm{~mL}$ could be generated. For each volume, four different expiratory flow profiles could be used with peak expiratory flow being attained between $21 \%$ and $63 \%$ of the total expiratory time. Two of these profiles, representing the lowest volume with an early peak tidal expiratory flow and the highest volume with a late peak tidal expiratory flow, are shown in figure 2. Respiratory frequency was kept constant at $30 \mathrm{~min}^{-1}$ for all measurements.

\section{Equipment for tidal breathing measurements}

The two systems selected for the purpose of this study were the well established and widely used SensorMedics 2600 (Sensormedics, Anaheim, CA, USA) [10-12] and a more recently developed, custom-made equipment based on the flow-through technique [1]. The SensorMedics 2600 (SM 2600) uses a screen pneumotachograph (PNT) with a measuring range of $\pm 10 \mathrm{~L} \cdot \mathrm{min}^{-1}$ and a resistance (RPNT) of $0.5 \mathrm{kPa} \cdot \mathrm{L}^{-1} \cdot \mathrm{s}$ as defined by the back pressure at $5 \mathrm{~L} \cdot \mathrm{min}^{-1}$ (Hans Rudolph Inc., Wyandotte, MI, USA). The flow-through technique (FTT), in which the face mask and both pneumotachographs $\left(\mathrm{PNT}_{1}\right.$, and $\left.\mathrm{PNT}_{2}\right)$ are continuously rinsed by a constant background flow that virtually eliminates apparatus dead space was developed to allow long-term measurements even in oxygen dependent infants [13]. Full details of previous validation of the FTT have been published previously $[1,14]$. The FTT consists of two screen PNTs with a measuring range of $\pm 24 \mathrm{~L} \cdot \mathrm{min}^{-1}$ with RPNT of $0.2 \mathrm{kPa} \cdot \mathrm{L}^{-1} \cdot \mathrm{s}$ as defined by the back pressure at $5 \mathrm{~L} \cdot \mathrm{min}^{-1}$ (Baby PNT Jaeger, Würzburg, Germany). The background flow (room air, $22^{\circ} \mathrm{C}$ ) was adjusted by a laboratory rotameter (Aalborg Instruments and Controls Inc., Orangeburg, NY, USA) to $6 \mathrm{~L} \cdot \mathrm{min}^{-1}$ and kept constant for all measurements. This background flow did not have any visible effect on measurements obtained with the SM 2600 when the two devices were connected to the pump via the T-piece for simultaneous measurements (fig. 1).

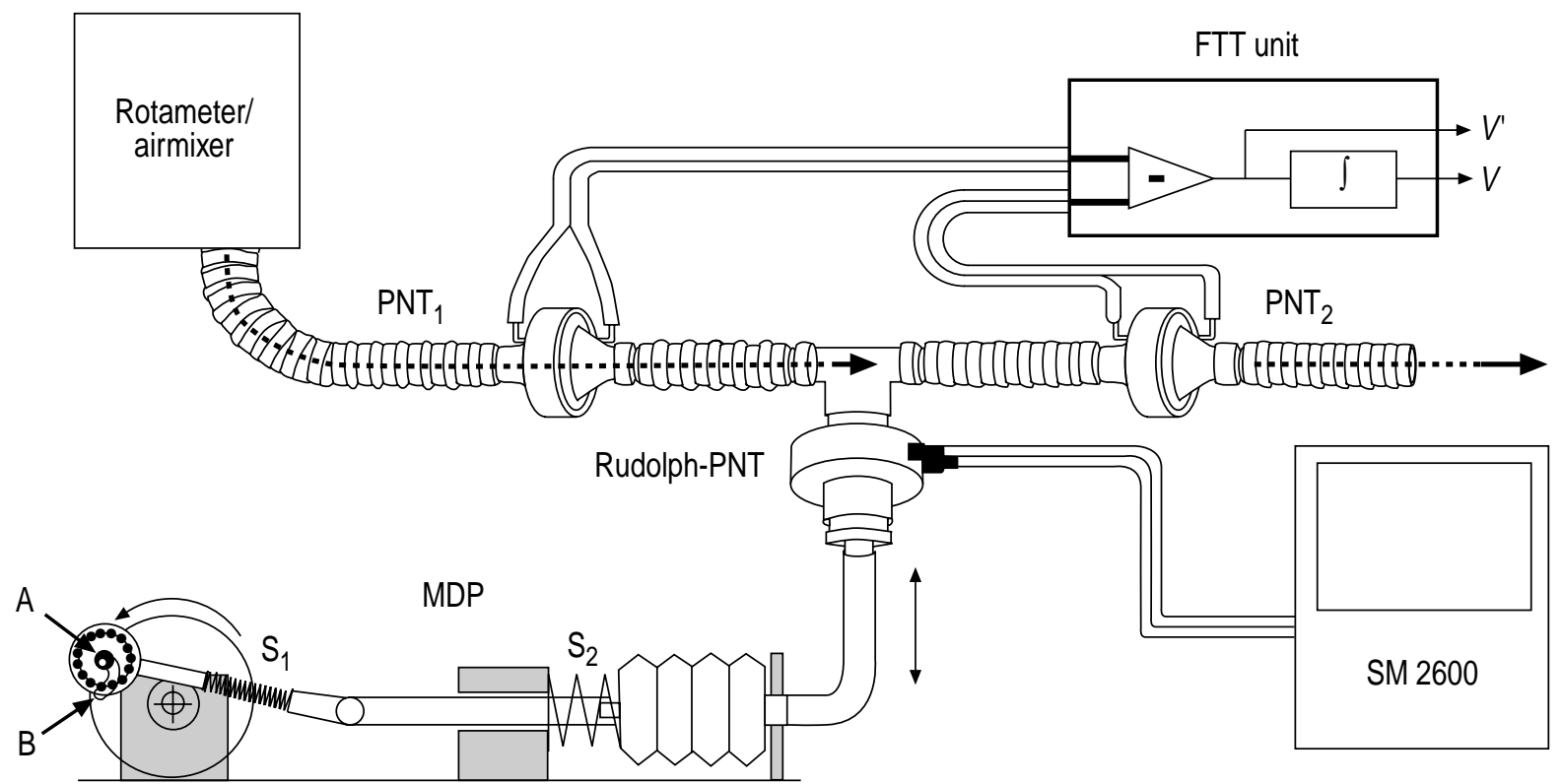

Fig. 1. - Equipment to validate accuracy of tidal breathing parameters by simultaneous measurements with the SensorMedics 2600

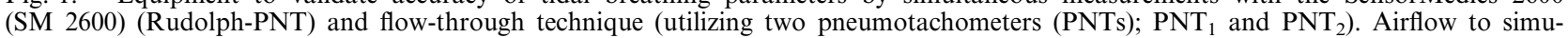
late the breathing pattern is generated by a motor driven pump (MDP) and a cam disc. The spring, $\mathrm{S}_{1}$, provided continuous guidance of a ball bearing moving along the cam with two different end-points, A and B. Different flow profiles could be generated by changing the cam disk. $V$ : minute ventilation; $V$ : volume; FTT unit: flow through technique measuring unit, measuring and integrating ( () the differences between $\mathrm{PNT}_{1}$ and $\mathrm{PNT}_{2}$. 

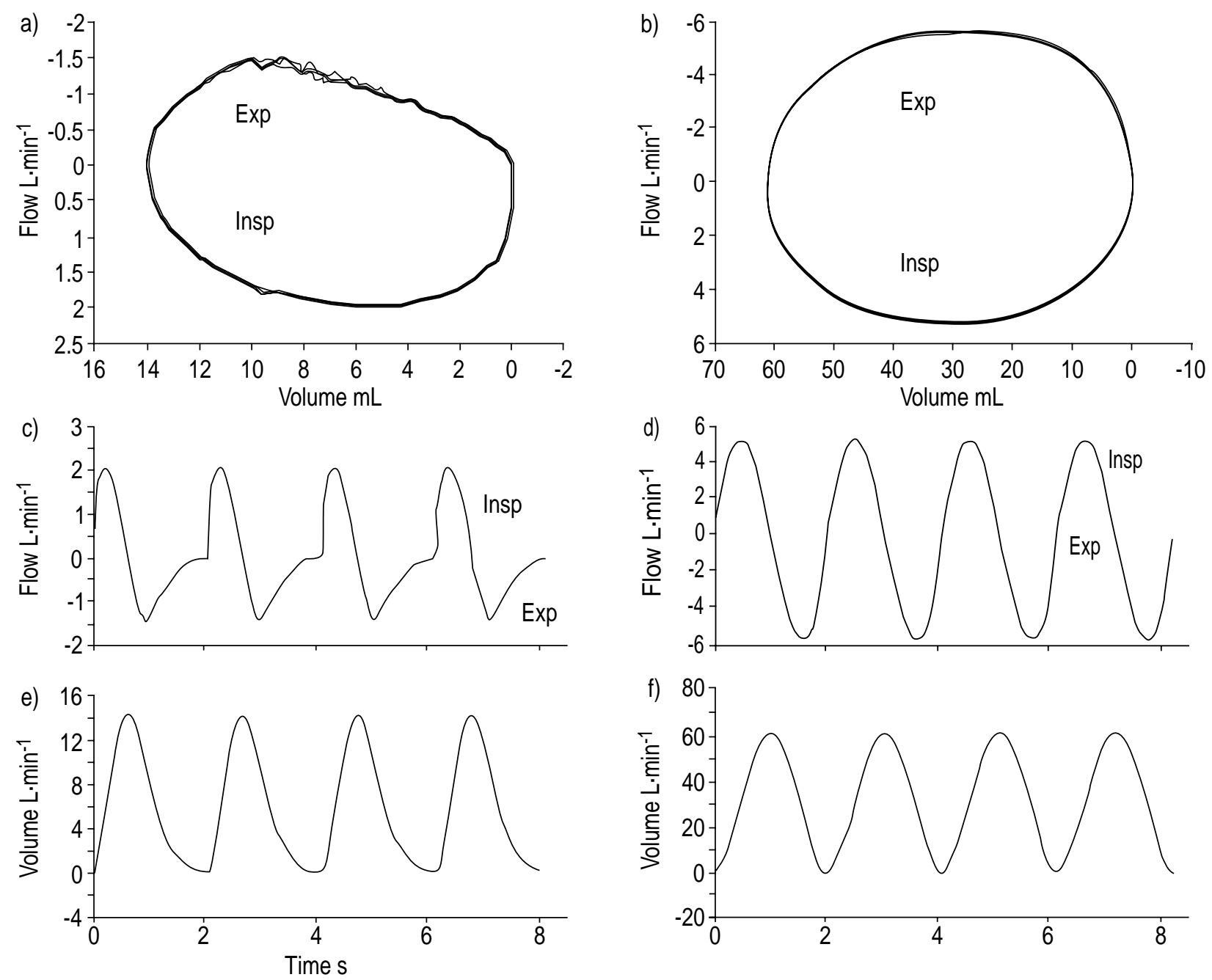

Fig. 2. - Example of recordings from four consecutive breathing cycles from the two different flow profiles (a, c and e represent flow profile 1 and $\mathrm{b}$, $\mathrm{d}$ and $\mathrm{f}$ represent flow profile 2) that were used for manual evaluation of the tidal breathing parameters. Collated flow-volume loops ( $\mathrm{a}$ and $\mathrm{b}$ ) and corresponding time based traces of flow (c and d) and volume (e and f) are shown for each profile. Insp: inspiration; Exp: expiration.

Both devices allowed raw flow transducer signals to be recorded as an electrical voltage signal. For the SM 2600 , this was achieved via a special service port of the device, whereas it was an integral part of the FTT system. There was comparable resolution of signal processing of the flow signals from both devices, with respect to both sampling rate $(256 \mathrm{~Hz}$ for the SM 2600 and $200 \mathrm{~Hz}$ for the FTT) and A/D conversion (14 and $16 \mathrm{Bit} \mathrm{A} / \mathrm{D}$ conversion, respectively). A warm up period of $30 \mathrm{~min}$ was allowed for both devices and calibration of the PNT, including the linearization procedure of the SM 2600, was performed prior to the measurements.

\section{Protocol}

For the evaluation, three tidal breathing parameters which depend only on the depth and frequency of the breathing cycle (tidal volume $(V \mathrm{~T})$, respiratory frequency $(f \mathrm{R})$, minute ventilation $\left(V^{\mathrm{E}} \mathrm{)}\right)$ were selected, together with four parameters which depend on the shape of the time based flow signal or tidal breathing flow-volume loop (TBFVL) (expiratory time $(t \mathrm{E})$, time to peak tidal expiratory flow as a proportion of tidal expiratory time $t \mathrm{PTEF} / \mathrm{tE}$, volume to peak tidal expiratory flow as a proportion of tidal volume $(V \mathrm{PTEF} / V \mathrm{~T})$ and flow when $25 \%$ of tidal volume remains in the lung (TEF25)).

Using the signals shown in figure 2, attempts were made to validate the automatically calculated parameters by comparing them with those calculated manually from appropriate print outs. This was relatively simple for the FTT as the calibrated flow and volume signals could be printed out on a large enough scale to permit accurate manual evaluation (fig. 2). Since this was not possible for the SM 2600 due to the relatively poor quality of the graphics, the tidal breathing parameters calculated by the SM 2600 were compared with those derived by manual evaluation of identical breaths that were measured and printed by the FTT. In addition, the uncalibrated raw flow signals of the SM 2600 were used to compare the values of timing 


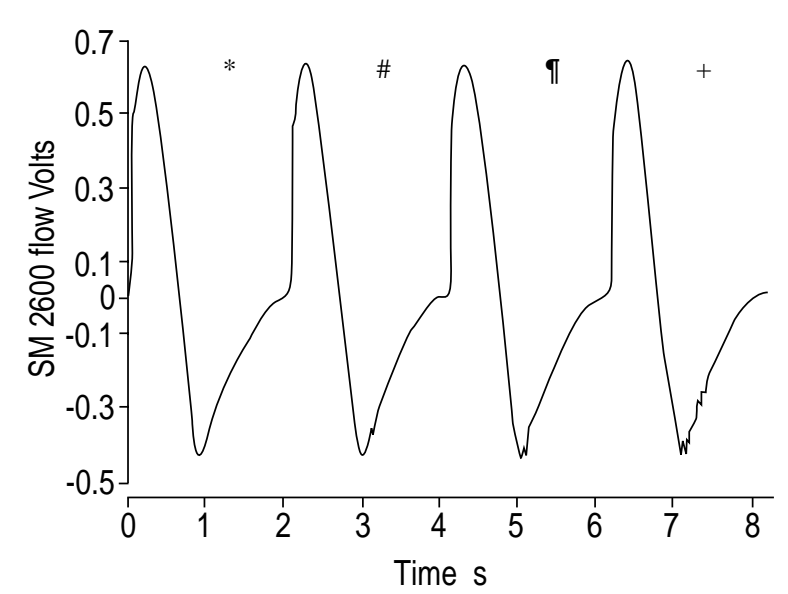

Fig. 3. - Comparison between values of expiratory time $(t \mathrm{E})$ and time to peak tidal expiratory flow as a proportion of $t \mathrm{E} t \mathrm{PTEF} / \mathrm{tE}$; T) that were evaluated manually from the uncalibrated raw flow signal from the SM 2600, measured in volts via a service port, and those displayed by the SM 2600 after automatic calculations on the same breaths (data in brackets). Positive voltage indicates respiration, negative indicates expiration. *: $t \mathrm{E}=42 \mathrm{~s}(1.40 \mathrm{~s})$, $t \mathrm{PTEF} / \mathrm{t}=20.6 \% \quad(11 \%) ; \quad \#: \quad t \mathrm{E}=1.42 \mathrm{~s} \quad(1.39 \mathrm{~s}), \quad t \mathrm{PTEF} / \mathrm{t} \mathrm{E} 21.4 \%$ $(11 \%)$; $t \mathrm{E}=1.42 \mathrm{~s}(1.37 \mathrm{~s}), t \mathrm{PTEF} / \mathrm{tE} 21.4 \%(11 \%) ;{ }^{+}: t \mathrm{E}=1.42 \mathrm{~s}$ $(1.40 \mathrm{~s}), t \mathrm{PTEF} / t \mathrm{E} 20.6 \%(13 \%)$.

parameters such as $t \mathrm{E}$ and $t \mathrm{PTEF} / t \mathrm{E}$ that were calculated both manually and automatically by the SM 2600 (fig. 3).

Since the SM 2600 only permits storage of four consecutive breathing cycles, precise matching of exactly the same breaths recorded by two different devices is extremely difficult. In an attempt to minimize this potential source of error, 12 breathing cycles were evaluated for each flow profile (12 consecutive cycles in FTT; $3 \times 4$ consecutive cycles with the SM 2600). The absolute and relative deviation between both devices was calculated as:

$$
\Delta \mathrm{X}=\mathrm{X}_{\mathrm{SM} 2600}-\mathrm{X}_{\mathrm{FTT}}
$$

and

$$
\delta \mathrm{X}[\%]=200 \frac{\mathrm{X}_{\mathrm{SM} 2600}-\mathrm{X}_{\mathrm{FTT}}}{\mathrm{X}_{\mathrm{SM} 2600}+\mathrm{X}_{\mathrm{FTT}}}
$$

respectively.

\section{Statistical methods}

Mean \pm SD were calculated for all ventilatory parameters and the comparability of parameters between the two devices was assessed using the method of BLAND and ALTMAN [15]. The coefficient of variation $(\mathrm{CV}(\%)=100 \times(\mathrm{SD} / \mathrm{mean}))$ was used to describe the reproducibility of each parameter and the CVs for each device were compared by a rank test. Statistical evaluations were performed using the software STATGRAPHICS (Vers. 4.0, Manugistics Inc., Rockville, MD, USA). A level of statistical significance of $p<0.05$ was accepted.

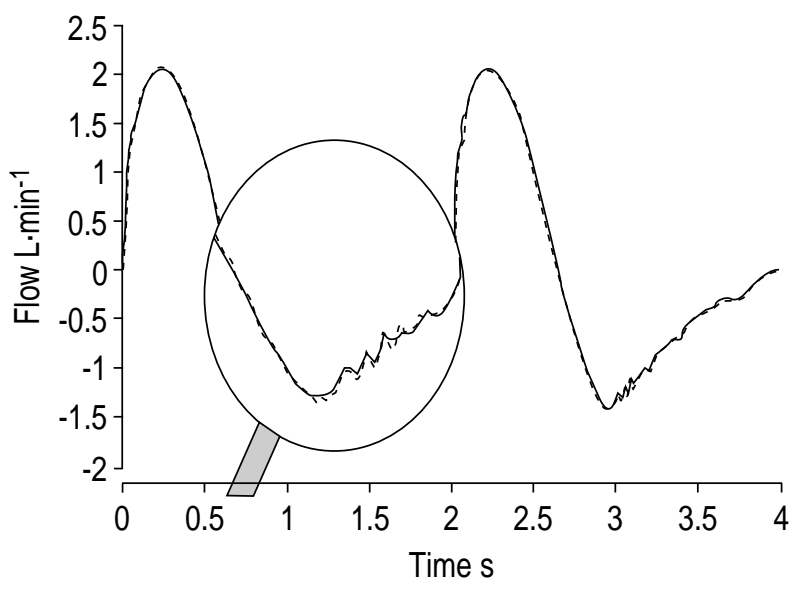

Fig. 4. - Example of flow signals of the SensorMedics 2600 (SM 2600) and flow-through technique (FTT) after adjustment of zero line and magnitude. - - - : SM 2600; — : FTT.

\section{Results}

The raw flow signals of the two devices differed in amplitude and zero off-set, but once these had been adjusted, there were no visible differences in the shape of the flow signal between the devices. A representative example of the flow signal simultaneously recorded by the two systems is shown in figure 4. The magnified portion of the trace shows that the background flow caused only a very slight increase in noise on the FTTsignal.

Comparison of automatically derived parameters with those calculated manually.

Table 1 shows the comparison of the values obtained for various tidal breathing parameters using the signals shown in figure 2, when evaluated manually, and when derived automatically by the two devices. As shown during previous evaluations [16], there was close agreement for all parameters between the results derived from manual evaluation and those automatically calculated by the FTT, with no bias between the two approaches. The deviations were always $<1.2 \%$ and could simply be attributed to reading errors. Similarly, for both flow profiles, there were only small deviations between values for $V_{\mathrm{T}}, V^{\prime} \mathrm{E} f \mathrm{R}, t \mathrm{E}$ and TEF 25 measured automatically by the SM 2600 and those evaluated manually $(<5 \%)$ (table 1$)$. By contrast, very marked and highly significant differences between these two approaches were observed for both $t \mathrm{PTEF} / \mathrm{tE}$, which was up to $61 \%$ lower, when calculated by the SM 2600 , than suggested from the manual evaluation of the trace, and $V \mathrm{PTEF} / V \mathrm{~T}$, which was up to $26 \%$ lower. These errors were most marked for flow profile 1, which had the smallest tidal volume, but remained significant (on average $17 \%$ lower) for $t \mathrm{PTEF} / t \mathrm{E}$ even when a tidal volume of $60 \mathrm{~mL}$ was used (flow profile 2). The discrepancies in $t \mathrm{PTEF} / \mathrm{t}$ e can be inspected on a breath by breath basis in figure 3 . This shows that, even when using the raw flow signal of the SM 2600, large deviations occur between the automatically determined $t \mathrm{PTEF} / \mathrm{t}$ E and that evaluated manually, and suggests that 


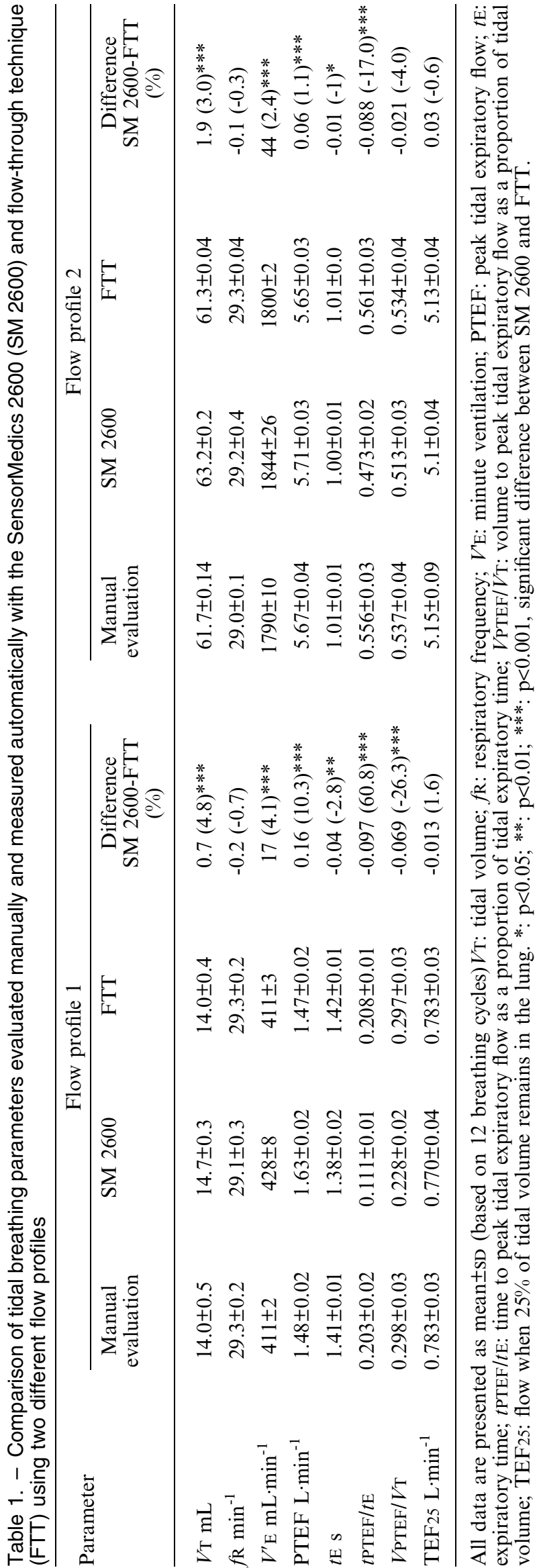

the large deviations in $t \mathrm{PTEF} / t \mathrm{E}$ and $V \mathrm{PTEF} / V \mathrm{~T}$ summarized in table 1 were probably caused by an error introduced at the signal processing level.

\section{Comparison between the devices}

As shown in table 2 , there was very close agreement (median deviation $<1.5 \%$ ) between the FTT and SM 2600 for those parameters which depended simply on the respiratory frequency and the magnitude of the flow and volume signals. For these parameters, there was no statistically significant influence of the flow profile or the magnitude of volume delivered on the differences between the devices,

By contrast, the values displayed by the SM 2600 for parameters which depend on the shape of the flow profile, especially $t \mathrm{PTEF} / t \mathrm{E}$ and $V \mathrm{PTEF} / V \mathrm{~T}$, differed considerably from the FTT results, with mean differences of up to $60 \%$ between the techniques for $t \mathrm{PTEF} / \mathrm{tE}$. Furthermore, for both of these parameters, the magnitude of the flow signal significantly influenced the extent to which values from the two devices differed; the lower the flow, the higher the discrepancy (table 2). Alterations in the flow profile however, did not have any significant effect on the magnitude of recorded differences.

The variability of the calculated parameters was very low for $V \mathrm{~T}, f \mathrm{R}, V^{\prime} \mathrm{E}, t \mathrm{E}$ and $\mathrm{TEF} 25$ (median $\mathrm{CV}<1.5 \%$ ), although the CVs for the SM 2600 where generally significantly higher than those for the FTT (table 3). Considerably larger CVs were found for $t \mathrm{PTEF} / t \mathrm{E}$, and $V \mathrm{PTEF} / V \mathrm{~T}$ for both devices.

\section{Discussion}

Whether using infant RFTs for clinical or research purposes, the accuracy of the devices used must be taken into account $[1,17]$. This is particularly pertinent for RFTs in sick newborns, in whom many difficulties in assessing respiratory function remain unresolved [18]. In the past, it has been extremely difficult to undertake such evaluations due to the lack of suitable lung models that can simulate an appropriate range of flow profiles [9], and the difficulties in obtaining details of algorithms, or in exporting either raw or calibrated signals from commercially available devices to facilitate such investigations [7]. The current study was able to address at least some of these difficulties, and provided clear evidence of the existence of discrepancies between equipment currently available to assess tidal breathing in infants.

\section{Comparison of both devices}

The SM 2600 is currently the most frequently used and extensively investigated commercial device for RFT in newborns [10-12], and hence was selected for this comparative study. In addition, during a recent assessment of the influence of apparatus deadspace on tidal breathing parameters in infants [1], marked discrepancies were identified in certain parameters over and above those that would be anticipated for physiological reasons, which warranted further investigation. 
Table 2. - Relative deviations in the tidal breathing parameters between SensorMedics 2600 (SM 2600) and flow-through technique (FTT) in relation to the volume of the applied signal

\begin{tabular}{|c|c|c|c|c|}
\hline \multirow{2}{*}{ Parameter } & \multicolumn{4}{|c|}{ Per cent difference between SM 2600 and FTT } \\
\hline & Volume $=15 \mathrm{~mL}$ & Volume $=28 \mathrm{~mL}$ & Volume $=46 \mathrm{~mL}$ & Volume $=60 \mathrm{~mL}$ \\
\hline$\delta V \mathrm{~T}$ & $2.7(-0.9-6.3)$ & $1.9(-1.7-5.5)$ & $1.8(-1.9-5.4)$ & $1.2(-2.3-4.9)$ \\
\hline$\delta f \mathrm{R}$ & $-0.03(-0.7-0.6)$ & $0.6(-0.1-1.2)$ & $-0.2(-0.9-0.4)$ & $0.1(-0.5-0.8)$ \\
\hline$\delta V^{\prime} \mathrm{E}$ & $2.7(-0.7-6.0)$ & $2.4(0.9-5.8)$ & $1.5(-1.8-4.9)$ & $1.4(-2.0-4.7)$ \\
\hline$\delta t \mathrm{E}$ & $-4.5(-7.2--1.8)^{*}$ & $-4.3(7.0-1.5)^{*}$ & $-2.4(-5.2-0.3)$ & $-2.0(-4.7-0.7)$ \\
\hline$\delta t \mathrm{PTEF} / t \mathrm{E}$ & $-58.5(-73.0--43.8) * * *$ & $-32.5(-47.1--17.9)^{* * *}$ & $-22.2(-36.8--7.6)^{* *}$ & $-24.8(-39.4-10.2)^{* *}$ \\
\hline$\delta V \mathrm{PTEF} / V \mathrm{~T}$ & $-30.5(44.4-16.5)^{* * *}$ & $-15.0(-28.9-1.0)^{*}$ & $-6.6(-20.5-7.3)$ & $-11.8(-25.7-2.1)$ \\
\hline$\delta \mathrm{TEF} 25$ & $-4.7(11.7-2.3)$ & $-5.3(-12.3-1.7)$ & $-3.7(-10.7-3.3)$ & $-1.7(-8.7-5.3)$ \\
\hline
\end{tabular}

Data presented as mean (95\% confidence interval) \% difference between SM 2600 and FTT (SM 2600-FTT). Abbreviations as in table 1. *: $\mathrm{p}<0.05 ; * *: \mathrm{p}<0.01 ; * * *: \mathrm{p}<0.001$, significant difference between SM 2600 and FTT.

Previous investigations have demonstrated that both the SM 2600 and the FTT can measure volume accurately $(<3 \%$ error) over the range of $10-60 \mathrm{~mL}[14,18]$, and this was confirmed in the present study. However, there is no published information about the accuracy of shape dependent tidal breathing parameters such as $t \mathrm{PTEF} / t \mathrm{E}$ which are widely used but much more difficult to evaluate.

The accuracy of the software in the FTT was relatively easy to validate, in that not only was there full access to the algorithms used, and the ability to export and inspect raw and calibrated signals such as ASCII files, but there was sufficient resolution in the graphics and printouts to enable accurate manual checks to be performed for the parameters that were automatically displayed by the device (figs. 2 and 3 ). However, since this was not possible with the SM 2600 due to the poor quality graphical printouts, detection of any flow-dependent measuring errors in $t \mathrm{PTEF} / \mathrm{tE}$ and $V \mathrm{PTEF} / V \mathrm{~T}$ would have been much more difficult. The equipment and software of the SM 2600 (which is currently undergoing major review) is at least $10 \mathrm{yrs}$ older than those of the FTT and significant technological progress in data processing and graphical display has occurred during this period. Nevertheless, the ability to access the raw flow signal from the SM 2600 via a special service port did allow some manual checks to be performed. Both devices have nearly the same flow resolution with regard to magnitude and time and, after numerical adjustments of the zero line and amplitude, it is evident that both raw flow signals are

Table 3. - Coefficients of variation for various tidal breathing parameters

Coefficient SensorMedics 2600 Flow-through technique of variation

\begin{tabular}{lll}
\hline$V_{\mathrm{T}}$ & $0.5(0.2-1.8)$ & $0.1(0.1-0.3) * * *$ \\
$f \mathrm{R}$ & $1.3(0.1-1.7)$ & $0.2(0.0-0.4)$ \\
$V^{\prime} \mathrm{E}$ & $0.9(0.2-1.8)$ & $0.2(0.1-0.4)^{* * *}$ \\
$t \mathrm{E}$ & $1.1(0.4-4.6)$ & $0.3(0.2-0.5)^{* * *}$ \\
$t \mathrm{PTEF} / t \mathrm{E}$ & $5.3(1.6-20.5)$ & $4.8(0.7-10.6)$ \\
$V \mathrm{PTEF} / V \mathrm{~T}$ & $6.6(1.6-20.5)$ & $7.5(1.0-10.3)$ \\
$\mathrm{TEF} 25$ & $1.4(0.6-6.7)$ & $1.2(0.4-2.0)$ \\
\hline
\end{tabular}

Results are displayed as median (range) for 12 consecutive breathing cycles. $* * *: p<0.001$ for comparison of variability between flow-through technique and SensorMedics 2600 . Abbreviations as in table 1 . virtually the same (fig. 4), Since there is no visible difference in the flow signals between the two devices, the observed differences in the displayed results must be the result of: 1) differences in the calibration factors; 2) numerical corrections (e.g. correction to body temperature, pressure, saturated (BTPS) conditions); 3) compensation and control of zero-line offset; or 4) the evaluation software.

\section{Deviations in the measured parameters}

The comparative in vitro measurements showed that, for parameters such as $V \mathrm{~T}, f \mathrm{R}$ and $V^{\prime} \mathrm{E}$, the observed differences between both devices were low over the whole volume range, and would not have any influence on the comparability of these parameters. By contrast, much larger deviations were found for tidal breathing parameters which depend on the shape of the flow and volume signal. Such parameters are much more influenced by signal processing (sample rate, analogue/ digital conversion, filtering), noise, software and to a large extent, by the shape of the flow profile. The curvature of peak values on the flow signal is often relatively flat such that small disturbances in the flow signal can cause large deviations in $t$ PTEF or VPTEF. This is reflected in the current study by the much higher $\mathrm{CVs}$ for $t$ PTET and $V \mathrm{PTEF}$ than for $V \mathrm{~T}, f \mathrm{R}$ and $V^{\prime} \mathrm{E}$ (table 3).

Many of the observed deviations between the FTT and SM 2600 (tables 1 and 2) may have resulted from the use of different algorithms to detect the beginning of inspiration and expiration. As discussed in detail recently, this is an extremely complex area which can easily lead to errors, especially in the presence of low flows or noisy signals $[19,20]$. For the more recently developed FTT, the equipment and software had been designed to ensure optimal stabilization of the endexpiratory level [16] and breath detection, even in the presence of noisy signals [19]. Since the SM 2600 is based on unpublished algorithms, it is difficult to identify the source of the discrepancies in $t \mathrm{PTEF} / \mathrm{tE}$ and $V \mathrm{PTEF} / V \mathrm{~T}$, between the two devices. The fact that there was close agreement in the values of $t \mathrm{E}$ calculated automatically, whether compared with those derived manually from the SM 2600 flow signal, or reported by the FTT, suggests that the error must lie in the way in which $t$ PTEF is automatically calculated by the SM 2600 . 


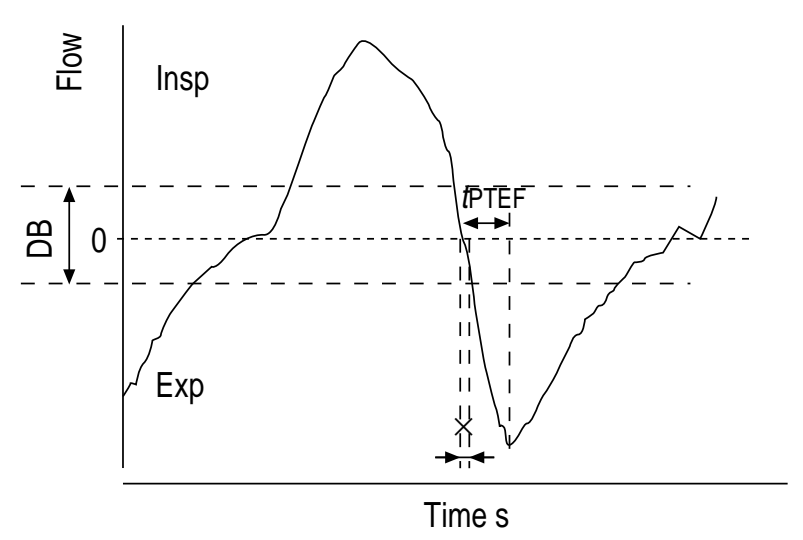

Fig. 5. - Illustration of how the time to peak tidal flow ( $t$ PTEF) may be underestimated if a dead band is used around zero flow to reduce the volume drift. With increasing magnitude of the flow signal the measuring errors caused by such a dead band will decrease. DB: dead band; Insp: inspiration; Exp: expiration; $x$ : time delay due to dead band.

In the current study, the deviations of $t \mathrm{PTEF} / \mathrm{tE}$ and $V \mathrm{PTEF} / V \mathrm{~T}$ between the two devices were found to decrease with increasing magnitude of the flow and volume signal (table 2). This dependence on flow for accuracy of displayed parameters often occurs in devices which use a dead band around the "zero flow" to stabilize the numerical integration of the signal (fig. 5). In such a system, all flow values within the selected dead band are set to zero so that there is no volume drift when flow is in this range, as may occur for example during a long expiratory pause. As shown in figure 5, such a dead band will have only a minimal influence on $f \mathrm{R}$ or $t \mathrm{E}$ but, as a result of the delay in recognizing start of expiration, may lead to significant underestimation of $t$ PTEF, the magnitude of which will be inversely proportional to that of the flow signal. Similar effects will be obtained if thresholds of the volume signal are used to reduce the drift of the displayed volume. Further potential sources of measurement error when calculating $t \mathrm{PTEF} / t \mathrm{E}$ and $V \mathrm{PTEF} / V \mathrm{~T}$ include failure to interpolate when identifying the exact moment of zero crossing between inspiration and expiration, and the presence of a zero-offset on the flow signal. The latter may arise from an undetected or poorly corrected off-set on the flow transducers or as the result of inappropriate correction of a volume drift.

The deviations of $t \mathrm{PTEF} / \mathrm{tE}$ found in this in vitro study were remarkably similar to those reported recently during consecutive measurements of tidal breathing in 86 infants using the same two devices [1]. In that study, paired measurements of $t \mathrm{PTEF} / \mathrm{tE}$ were on average $54 \%$ lower when measured with the SM 2600 than with the FTT. When using similar volumes and flow profiles in this in vitro study, the relative underestimation was $59 \%$. This suggests that, in contrast to the differences between the two devices for $V \mathrm{~T}$ and $f \mathrm{R}$ during in vivo studies, which can simply be attributed to changes in breathing pattern in response to the apparatus deadspace, the observed discrepancies in timing parameters such as $t \mathrm{PTEF} / t \mathrm{E}$ during such studies may be almost entirely due to differences in signal processing.

\section{Clinical implications}

The SM 2600 represents the first generation of commercial equipment for infant respiratory function testing, and has made an important contribution to the clinical use of RFT in infancy. An unexpected finding from this study was that the measuring errors of the older device were not due to limitations of the sensors/ transducers, but more to limitations in the software that was available at this time. Inability to perform simple checks on the accuracy of displayed parameters due to poor graphical resolution of the displayed and printed signals, together with the lack of suitable means of assessing performance formally, meant that any errors could easily go undetected for long periods of time. Since evaluations of this nature have rarely been performed, similar problems may well exist in other currently available systems, whether commercially available or custom built.

The results from this study suggest that equipment for infant RFTs needs to be carefully checked using a suitable model [9] before clinical application [4, 6, 18]. When measuring tidal breathing, information about the accuracy of volume measurements alone is not sufficient, since the accuracy of tidal breathing parameters depends on measuring both the range and the shape of the flow signals. Extensive model measurements for infant RFT using different flow signal patterns have not been performed, and any previous evaluations have been based on relatively large sinusoidal signals that are rarely observed during clinical measurements.

The discrepancies in $t \mathrm{PTEF} / \mathrm{tE}$ during both in vitro and in vivo studies were so large that values reported in the literature collected using different devices will clearly not be comparable. Furthermore, the relationship between the magnitude of error and absolute flows may seriously impair interpretation of the effects of disease, therapeutic interventions or growth and development on such parameters. There is therefore an urgent need for the user to have access to the algorithms and thresholds that are used in any specific device, and to understand how differences between devices may influence the results.

During recent years, there have been dramatic technological advances in both computer hardware and software. This has been accompanied by an increased commitment from both users and manufacturers to establish internationally approved standards in order to improve the confidence with which such equipment can be used [6, 7]. Indeed, following recent discussions with the European Respiratory Society/American Thoracic Society task force on standardization of infant respiratory function tests, manufacturers have agreed that in future, details of relevant algorithms will be more readily available to the users. This would greatly facilitate meaningful evaluations and comparisons of different systems, and hopefully minimize the occurrence of the type of discrepancies identified in this study.

\section{References}

1. Schmalisch G, Foitzik B, Wauer R, Stocks J. The influence of apparatus deadspace on tidal breathing 
parameters in newborns: comparison of the "flowthrough" and conventional techniques. Eur Respir $J$ 2001; 17: 108-114.

2. Cunningham MD, Shook LA. A comparison of intensive care monitoring systems for pulmonary dynamics of preterm infants undergoing mechanical ventilation. In: Bhutan VK, Shaffer TH, Vidyasagar D, eds. Neonatal pulmonary function testing: Physiological, technical and clinical considerations. Ithaea, Perinatology Press; 1988; 47-59.

3. Hauschild M, Schmalisch G, Wauer RR. Measuring accuracy of commercial respiratory function equipment and ventilation monitors for newborn infants. Klin Padiatr 1994; 206: 167-174.

4. Jackson EA, Coates AL, Gappa M, Stocks J. In vitro assessment of infant pulmonary function equipment. Pediatr Pulmonol 1995; 19: 205-213.

5. Roske K, Foitzik B, Wauer RR, Schmalisch G. Accuracy of volume measurements in mechanically ventilated newborns: a comparative study of commercial devices. J Clin Monit Comput 1998; 14: 413-420.

6. Frey U, Stocks J, Coates AL, Sly PD, Bates JH. Specifications for equipment used for infant pulmonary function testing. Eur Respir $J$ 2000; 16: 731740.

7. Frey U, Stocks J, Sly PD, Bates JH. Specification for signal processing and data handling used for pulmonary function testing. Eur Respir J 2000; 16: 10161023.

8. Stocks J, Sly PD, Tepper RS, Morgan WJ. Infant respiratory function testing. New York, Wiley-Liss 1996.

9. Frey U, Reinmann B, Stocks J. A standardized mechanical infant lung model to test infant lung function equipment. Am J Respir Crit Care Med 1999; 159: A478.

10. Lodrup Carlsen KC, Magnus P, Carlsen KH. Lung function by tidal breathing in awake healthy newborn infants. Eur Respir J 1994; 7: 1660-1668.

11. Emralino F, Steele AM. Effects of technique and ana- lytic conditions on tidal breathing flow volume loops in term neonates. Pediatr Pulmonol 1997; 24: 86-92.

12. Carlsen KCL, Stenzler A, Carlsen KH. Determinants of tidal flow volume loop indices in neonates and children with and without asthma. Pediatr Pulmonol 1997; 24: 391-396.

13. Schmalisch G, Wauer RR, Böhme B. Changes in pulmonary function in preterm infants recovering from RDS following early treatment with Ambroxol: Results of a randomized trial. Pediatr Pulmonol 1999; 27: 104-112.

14. Foitzik B, Schmalisch G, Wauer RR. Effect of background flow on the accuracy of respiratory flow and respiratory volume measurement in newborn infants. Biomed Tech Berl 1995; 40: 282-288.

15. Bland JM, Altman DG. Statistical methods for assessing agreement between two methods of clinical measurement. Lancet 1986; 1: 307-310.

16. Foitzik B, Schmidt M, Windstetter D, Wauer RR, Schmalisch G. Leak measurements in spontaneously breathing premature newborns by using the flowthrough technique - Special Communication. J Appl Physiol 1998; 85: 1187-1193.

17. Dundas I, Beardsmore C, Wellman T, Stocks J. A collaborative study of infant respiratory function testing. Eur Respir J 1998; 12: 944-953.

18. Schmalisch G, Wauer RR. Current technical limitations of lung function testing in ventilated infants and infants breathing spontaneously. Padiatr Grenzgeb 1995; 34: 21-37.

19. Schmidt M, Foitzik B, Wauer RR, Winkler F, Schmalisch G. Comparative investigations of algorithms for the detection of breaths in newborns with disturbed respiratory signals. Comput Biomed Res 1998; 31: 413-425.

20. Bates JHT, Schmalisch G, Filbrun D, Stocks J. Standards for infant respiratory function testing: Tidal breath analysis for infant pulmonary function testing. Eur Respir J 2000; 16: 1180-1192. 